\title{
Extracting Geospatial Entities from Wikipedia
}

\author{
Jeremy Witmer and Jugal Kalita \\ University of Colorado, Colorado Springs \\ Computer Science Department \\ 1420 Austin Bluffs Pkwy Colorado Springs, CO 80918 \\ jeremy@geografikos.net,kalita@eas.uccs.edu
}

\begin{abstract}
This paper addresses the challenge of extracting geospatial data from the article text of the English Wikipedia. In the first phase of our work, we create a training corpus and select a set of word-based features to train a Support Vector Machine (SVM) for the task of geospatial named entity recognition. We target for testing a corpus of Wikipedia articles about battles and wars, as these have a high incidence of geospatial content. The SVM recognizes place names in the corpus with a very high recall, close to $100 \%$, with an acceptable precision. The set of geospatial NEs is then fed into a geocoding and resolution process, whose goal is to determine the correct coordinates for each place name. As many place names are ambiguous, and do not immediately geocode to a single location, we present a data structure and algorithm to resolve ambiguity based on sentence and article context, so the correct coordinates can be selected. We achieve an f-measure of $82 \%$, and create a set of geospatial entities for each article, combining the place names, spatial locations, and an assumed point geometry. These entities can enable geospatial search on and geovisualization of Wikipedia.
\end{abstract}

\section{Introduction}

As the amount of unstructured user-generated content on the Internet increases, the need for methods to extract information from it also increases. We must refine tools and techniques for automatically extracting data in a machineunderstandable format. A significant number of queries on the Internet target geographical data, making this a good starting point. Google Trends ${ }^{1}$ data, and the increasing popularity of Google Maps ${ }^{2}$ indicate that queries for location data represent a significant fraction of search queries.

\footnotetext{
1 http: //www.google.com/trends/

${ }^{2}$ http://maps.google.com
}

Our work in this paper has two objectives. The first is to extract geospatial named entities (place names) from unstructured text documents, such as Wikipedia articles. For this research, we use only the article text of Wikipedia, ignoring the wiki and HTML markup. The second objective of the system is to further process the geospatial NEs, to resolve a correct (latitude, longitude) coordinate pair for the place name, in context of the article.

As an example, consider an excerpt from of an article on the Spanish-American War from Wikipedia given in Figure 1. In this article snippet, we have highlighted in green the geospatial named entities our system is able to find.

Our research in this area is motivated by a number of factors. The goal of the development of the Geografikos package is a software system that creates a database of Wikipedia articles in which each article has an associated structured set of geospatial entities, extracted from the article. This database will allow geospatial querying, information retrieval, and geovisualization to be applied to the Wikipedia articles. Further, we wish to open-source the Geografikos software on completion.

Section 2 of the paper discusses relevant background research. Section 3 focuses on using SVMs for extracting geospatial NEs from text. Section 4 discusses geocoding the extracted place names by situating them within a geographical unit in the world. Finally, we conclude with a discussion of possibilities for future research.

\section{Background Research}

Named entity recognition refers to the extraction of words and strings of text within documents that represent discrete concepts, such as names and locations. The term "Named Entity Recognition" describes the operations in natural language processing that seek to extract the names of persons, organizations, locations, other proper nouns, and numeric terms, such as percentages and dollar amounts. The term "Named Entity" was defined by the MUC-6, sponsored by DARPA in 1996 [5]. The NE recognition (NER) 


\section{Spanish-American War}

From Wikipedia, the free encyclopedia

(Redirected from Spanish American War)

The Spanish-American War was an armed military conflict between Spain and the United States that took place between April and August 1898, over the issues of the liberation of Cuba. The war began after American demand for the resolution of the Cuban fight for independence was rejected by Spain. Strong expansionist sentiment in the United States motivated the government to develop a plan for annexation of Spain's remaining overseas territories including the Philippines, Puerto Rico, and Guam. ${ }^{[3]}$

The revolution in Havana prompted the United States to send in the warship USS Maine to indicate high national interest. Tension among the American people was raised because of the explosion of the USS Maine, and the yellow journalist newspapers that accused the Spanish of oppression in their colonies, agitating American public opinion. The war ended after victories for the United States in the Philippine Islands and Cuba.

On December 10, 1898, the signing of the Treaty of Paris gave the United States control of Cuba, the Philippines, Puerto Rico, and Guam.

\section{Figure 1. Example Wikipedia Article with Geospatial NEs Highlighted}

task was further defined, and expanded to language independence by the Conference on Natural Language Learning shared task for the 2002 and 2003 conferences.

Numerous approaches have been tried since MUC-6 to increase performance in NER, including Hidden Markov Models, Conditional Random Fields, Maximum Entropy models, Neural Networks, and Support Vector Machines (SVM).

SVMs [14] are a subset of kernel-based methods used for regression and classification. An SVM views input data as feature vectors in $N$-dimensional space, with the goal of finding an optimal hyperplane that separates the vectors into positive and negative classes, based on a function learned from a set of training vectors. Theoretical and experimental results indicate that SVMs are able to generalize very well and avoid overfitting in high dimensional feature spaces.

SVMs have shown significant promise for the task of NER. Starting with the CoNLL 2002 shared task on NER, SVMs have been applied to this problem domain with improving performance. Bhole et al. have done significant work in extracting named entities from Wikipedia and relating them over time using SVMs [1]. In the biomedical domain, Takeuchi and Collier have demonstrated that SVMs outperform HMMs for NER [13]. Furthermore, Lee et al. [8] and Habib [6] have shown that SVMs are applicable to multi-class NER and text classification problems. Isozaki and Kazawa provide results in [7] showing that chunking and part-of-speech tagging also provide performance increases for NER with SVMs, both in speed and in f-measure. In [3], Dakka and Cucerzan demonstrate an SVM that achieves an f-measure of 0.954 for LOC entities in Wikipedia articles, and an f-measure of 0.884 across all NE classes. Using an SVM in conjunction with a fuzzy
SVM which allows greater margin overlap in the cost parameter, Mansouri et al. demonstrate excellent performance on NER [9]. Thus, research into text classification and NER has found that SVMs provide good performance on NER tasks, despite the inherent difficulties in NER.

\section{Geospatial Named Entity Extraction}

Our first task is to extract all candidate geospatial NEs. The desired output of this stage of the Geografikos package is a set of words and strings that may be place names. Below, we discuss the selection of the feature set used to to train the SVM, the generation of the training and testing corpuses, the preprocessing of the text, and finally, the results. In this initial NE recognition task we target high recall, even with a sacrifice in precision, as this means that we extracted all of the possible candidate NE strings.

\subsection{Training Corpus Generation}

We downloaded a number of previously tagged data sets to provide the training material for the SVM, along with other resources that required hand-tagging:

- Spell Checker-Oriented Word List (SCOWL) ${ }^{3}$ to provide a list of English words.

- CoNLL 2003 shared task dataset on multi-language NE tagging 4 containsing tagged named entities for PER, LOC, and ORG, in English.

\footnotetext{
${ }^{3}$ http: //downloads. sourceforge. net/wordlist/ scowl-6.tar.gz

${ }^{4}$ http: //www. cnts.ua.ac.be/conll2003/ner/
} 
- Reuters Corpus from NIST: ${ }^{5}$ 15,000 selected words from the corpus were hand-tagged for LOC entities.

- CoNLL 2004 shared task dataset, on Semantic Role Labeling ${ }^{6}$ tagged for English LOC NEs.

- CoNLL 2005 shared task dataset, on Semantic Role Labeling $^{7}$ tagged for English LOC NEs.

- Geonames database of 6.2 million names of cities, counties, countries, land features, rivers and lakes. ${ }^{8}$

To generate the training corpus, we combined our handtagged Reuters corpus and the three CoNLL datasets. We extracted those records in the 2.5 million word Geonames database in either the P or A Geonames codes (Administrative division and Population center), which provided the names of cities, villages, countries, states, and regions. Combined with SCOWL wordlist, which provided negative examples, this resulted in a training set of 1.2 million unique words.

\subsection{Testing Corpus Generation}

To generate the test corpus, we selected 90 articles from Wikipedia on battles and wars around the world. Before the text of each of the articles was tagged, the actual text returned from the database required preprocessing to remove the Wikipedia-specific markup, HTML, and other extraneous information. Preprocessing was in five steps.

First, extraneous sections such as "References", "See Also", or "Notes" were removed. These sections do not contribute any information to the primary text. Second, all HTML markup was stripped. While most markup in Wikipedia is done in wiki style, some markup in the pages is still done in HTML.

Third, the Wikipedia infoboxes in the page were processed. Infoboxes are special constructs that reference external blocks of text. A certain number of these infoboxes are system infoboxes conveying information for processing and display which were removed completely. However, if the infobox contributed to the primary text of the page, one of two operations were performed. In a small set of cases, the infoboxes were links to another full article page. These infoboxes were replaced with a Wikipedia link to the other page. For all the other infoboxes, the full text of the infobox was inserted into the primary text. Because infoboxes can also contain infoboxes, this processing was recursive.

Fourth, for Wikipedia-style links, the link text was removed, and replaced with either the name of linked page or with the caption text for links with a caption. Finally,

\footnotetext{
${ }^{5}$ http://trec.nist.gov/data/reuters/reuters.html

${ }^{6}$ http://www.lsi.upc.es/ srlconll/st04/st04.html

${ }^{7}$ http://www.lsi.upc.es/ srlconll/soft.html

${ }^{8}$ http: //WwW.geonames.org/export/
}

cleanup was done for any remaining link or infobox symbols. Links to external sites were removed. Special characters, unicode and special symbols were removed, as these did not add any information, and tended to cause exceptions and problems in string processing.

The cleaned primary text of each page was split into an array of individual words, and the individual words were hand-tagged as geospatial NEs or not, either 1 or 0.

\subsection{SVM Feature Selection}

To define the features describing the geospatial NEs, we drew primarily from the work done for word-based feature selection by Habib [6]. She used lexical, word-shape, and other language-independent features to train an SVM for the extraction of NEs in the biomedical domain. Furthermore, the work done by Cucerzan et al. [2] on extracting NEs from Wikipedia provided further features.

For feature set selection, we postulated that each word in the geospatial NE should be independent, and that the training corpus should be split into individual words. After testing various combinations of features, we used the following features for each word to generate the feature vectors for the SVM, with equal weighting on all features:

- Word length: alphanumeric character count, ignoring punctuation marks

- Vowel count, consonant count, and capital count in the word

- First capital: if the word begins with a capital letter

- If the word contains two vowels together

- If the word contains two identical consonants together

- If the word ends with a comma, a period, a hyphen, a semi-colon, or a colon

- If the word begins or ends with a quote

- If the word begins or ends with a parenthesis

- If the word is plural, or a single character

- If the word is alphanumeric, purely numeric, purely text, all uppercase, or all lowercase

- If the word has an English suffix or prefix

- If the word is a preposition, an article, a pronoun, a conjunction, an interrogative or an auxiliary verb

\subsection{SVM Training and Results}

We used LibSVM to train an SVM based on the feature vectors and two parameters, $C$ and $\gamma$, which are the cost and degree coefficient of the SVM kernel function. LibSVM provides a Python script to perform a step-wise grid search for the optimal $C$ and $\gamma$ values, finding local maxima in the fitness graph, and randomly jumping to a new $(C, \gamma)$ starting point to try to find the overall maximum. After using the grid search, the optimal parameters were determined to be $C=8$ and $\gamma=0.03125$. 
Table 1. Training Corpus Combination Results for the NER SVM

\begin{tabular}{||l|l|l|l||}
\hline Corpus & Precision & Recall & F \\
\hline R & 0.38 & 0.63 & 0.47 \\
R + C03 + C04 + C05 & 0.45 & 0.68 & 0.54 \\
R + C03 + C04 + C05 + & 0.49 & 0.75 & 0.59 \\
SCW & & & \\
Wiki & 0.65 & 0.34 & 0.44 \\
Wiki + R + C03 + C04 + & 0.60 & 0.55 & 0.57 \\
C05 + SCW & & & \\
Geo + R + C03 + C04 + & TC & TC & TC \\
C05 + SCW & & & \\
FGeo + R + C03 + C04 + & $\mathbf{0 . 5 1}$ & $\mathbf{0 . 9 9}$ & $\mathbf{0 . 6 7}$ \\
C05 + SCW & & & \\
\hline
\end{tabular}

R: Reuters Corpus. C03: CoNLL 2003 dataset. C04:

CoNLL 2004 dataset. C05: CoNLL 2005 dataset. Wiki:

Wikipedia data. SCW: SCOWL wordlist. Geo: full

Geonames database. FGeo: Filtered Geonames database. TC: Training Canceled.

We selected a radial basis function kernel, and applied it to the C-SVC, the standard cost-based SVM classifier. The SVM was trained using feature vectors based on the second training corpus, requiring 36 hours to complete. This trained SVM was used throughout the rest of this work.

Our goal was to obtain high recall while maintaining good precision. Table 1 shows the precision, recall, and $\mathrm{f}$ measure numbers for training corpuses created with different subsets of the training data. The performance numbers are the results from the Wikipedia testing corpus.

The recall from the SVM increased significantly when we introduced the Geonames database as part of the training corpus, but the full Geonames database, some 2.5 million words, significantly impacted the SVM, and we were unable to complete training on an SVM, after 5 days. In part, this was because the training set is large, but we also found that there were many occurrences of words marked both as positive and negative examples of geospatial NEs. We then filtered the Geonames database to the final set, using only administrative division and population center names, which brought the precision back up to acceptable levels.

After selecting the optimal training corpus, we optimized the feature set for the SVM. Table 2 shows a breakdown of the feature sets we tested separately. We found that the list presented earlier provided the best performance by close to $10 \%$ in f-measure over any other combination of features.

After selecting the final set of features, we performed some initial experiments in weighting the features differently. We found that in all cases, changing the weighting on the features resulted in an f-measure loss between $0.21 \%$ and $3.4 \%$, not a significant impact on the perfor-

\section{Table 2. Feature Set Combination Results for the NER SVM}

\begin{tabular}{||l|l||}
\hline Feature Set & F-Measure \\
\hline SHP+PUN+HYP+SEP & 0.44 \\
SHP+CAP+PUN+HYP+SEP & 0.47 \\
CAP+VOW+CON & 0.51 \\
SHP+CAP & 0.54 \\
SHP+CAP+VOW+CON & 0.56 \\
SHP+CAP+VOW+CON+AFF+POS & 0.58 \\
SHP+CAP+VOW+CON+PUN & \\
+HYP+SEP+AFF+ POS & $\mathbf{0 . 6 7}$ \\
\hline
\end{tabular}

SHP: Word shape, including length and alphanumeric content. CAP: Capital positions and counts. VOW: Vowel positions and counts. CON: Consonant positions and counts. PUN: Punctuation. HYP: Hyphens. SEP:

Separators, quotes, parenthesis, etc. AFF: Affixes. POS: part of speech categorization.

mance. We concluded that, we did not need to spend a significant amount of time on working with the feature weighting, and simply weighted all features equally at 1.0.

Once we found the optimal feature set for the SVM, we re-trained and tested the SVM with other corpus combinations. However, the results of the original corpus selection remained the best.

With this setup, we were successful in training the SVM to return high recall numbers, recognizing $99.8 \%$ of the geospatial named entities. Unfortunately, this was at the cost of precision. The SVM returned noisy results with only $51.0 \%$ precision, but the noise was processed out during the NE geocoding process, to obtain our final results, discussed in the next section.

\section{Geospatial Named Entity Resolution}

The SVM extracted a set of candidate geospatial NEs from the article text. For each candidate string, the second objective was to decide whether it was a geospatial NE, and to determine the correct (latitude, longitude), or $(\phi, \lambda)$ coordinate pair for the place name in context of the article.

To resolve the candidate NE, a lookup was made using Google Geocoder ${ }^{9}$. If the entity reference resolved to a single geospatial location, no further action was required. Otherwise, the context of the place name in the article, a new data structure and a rule-driven algorithm were used to decide the correct spatial location for the place name.

Our research targeted the so-called context locations, defined as "the geographic location that the content of a web

\footnotetext{
${ }^{9}$ http: //code.google.com/apis/maps / documentation/geocoding/index.html
} 
Table 3. Places With Multiple Names and Names Applied to More Than One Place in the Getty Thesaurus of Geographic Names

\begin{tabular}{||l|l|l||}
\hline Continent & $\begin{array}{l}\% \text { places } \\
\text { with multiple } \\
\text { names }\end{array}$ & $\begin{array}{l}\% \text { names } \\
\text { with multiple } \\
\text { places }\end{array}$ \\
\hline North \& Central & 11.5 & 57.1 \\
America & & \\
Oceania & 6.9 & 29.2 \\
South America & 11.6 & 25.0 \\
Asia & 32.7 & 20.3 \\
Africa & 27.0 & 18.2 \\
Europe & 18.2 & 16.6 \\
\hline
\end{tabular}

resource describes" [16]. Our task is close to that of word sense disambiguation, as defined by Cucerzan [2], only that we consider the geospatial context and domain instead of the lexical context and domain. Sehgal et al. demonstrate good results for geospatial entity resolution using both spatial (coordinate) and non-spatial (lexical) features of the geospatial entities [11]. Zong et al. demonstrate a rulebased method for place name assignment, achieving a precision of $88.6 \%$ on disambiguating place names in the United States, from the Digital Library for Earth System Education (DLESE) metadata [18].

While related to the work done by Martins et al. in [10], we apply an SVM for the initial NER task, and only use the geocoder/gazetter for lookups after the initial candidate geospatial NEs have been identified. We also draw on our earlier work in this area, covered in [17]

The primary challenge to the resolution and disambiguation is that multiple names can refer to the same place, and multiple places can have the same name. The statistics for these place name and reference overlap are given in Table 3 , from [12]. The table demonstrates that the largest area with ambiguous place names is North and Central America, the prime areas for our research. Most of the name overlaps are in city names, and not in state/province names. These statistics are further supported by the location name breakdowns on the individual Wikipedia articles in Table 4. The details in this table are broken down into countries, states, foreign cities, foreign features, US cities, and US features, where "features" are any non-state or city geospatial features, such as rivers, hills, and ridges. The performance of the Geografikos system on NER extraction and disambiguation is indicated. The Battle of Gettysburg, with over 50\% US city names, many of which overlap, resulted in the lowest performance. The Liberty Incident, in the Mediterranean Sea, with less than $10 \%$ US city and state names combined, showed significantly better performance.

\subsection{Google Geocoder}

We used Google Geocoder as the gazetteer and geocoder for simplicity, as much research has already been done in this area. Vestavik and D'Roza et al provide an excellent overview of existing technology in this area [15, 4]. Google Geocoder provides a simple REST-based interface that can be queried over HTTP, returning data in a variety of formats.

For each geospatial NE string submitted as a query, Google Geocoder returns 0 or more placemarks as a result. Each placemark corresponds to a single $(\phi, \lambda)$ coordinate pair. If the address string is unknown, or another error occurs, the geocoder returns no placemarks. If the query resolves to a single location, Google Geocoder returns a single placemark. If the query string is ambiguous, it may return more than one placemarks. A country code can also be passed as part of the request parameters to bias the geocoder toward a specific country. Google Geocoder returns locations at roughly four different levels, Country, State, City, and Street/Feature, depending on the address query string and the area of the world.

In the Geografikos package, the address string returned from Google Geocoder was separated into the four parts, and each part was stored separately, with the coordinates. The coordinates were also checked against the existing set of locations in the database for uniqueness. For this research, coordinates had to be more than 1/10th of a mile apart to be considered a unique location.

\subsection{Location Tree Data Structure}

The output from the Google Geocoder was fed thorough our location tree data structure, which, along with an algorithm driven by a simple set of rules, ordered the locations by geospatial division, and aided in the disambiguation of any ambiguous place name.

The location tree operated at four levels of hiearchy, Country, State/Province, City, and Street/Local Geographic Feature. Each placemark response from the geocoder corresponded to one of these geospatial divisions. A separate set of rules governed the insertion of a location at each level of the tree. For simple additions to the tree, the rules were:

1. If the location is a country, add it to the tree

2. If the location is a state or province, add it to the tree

3. If the location is a city in the US, and the parent node in the tree is a state, add it to the tree

4. If the location is a city outside the US, add it to the tree

5. If the location is a street or feature inside the US, and the parent node is a state or city, add it to the tree

6. If the location is a street or outside the US, and the parent node is either a country or city, add it to the tree 
Table 4. Place Name Details for Example Wikipedia Articles. All numbers are percentages.

\begin{tabular}{llllllll}
\hline Article name & Foreign features & US Features & Foreign Cities & US Cities & States & Countries & F-measure \\
\hline Battle of Gettysburg & 0 & 31 & 1 & 51 & 15 & 2 & 68.2 \\
War of 1812 & 3 & 18 & 5 & 30 & 8 & 36 & 81.6 \\
World War II & 20 & 0 & 17 & 1 & 0 & 62 & 87.0 \\
Liberty Incident & 25 & 1 & 10 & 6 & 1 & 57 & 90.5 \\
\hline
\end{tabular}

Rules 3 and 5 are US-specific because Google Geocoder has much more detailed place information for the US than other areas of the world, requiring extra processing.

Any location that did not match any rule was placed on a pending list. Each time a new location was added, the tree was re-sorted to ensure that the correct hierarchy was maintained, and each location on the pending list was rechecked to see if it now matched the rules for insertion.

If the location returned from Google Geocoder had a single placemark and matched the rules, it was placed in the tree. If the location returned corresponded to multiple coordinates, another set of calculations was required before running insertion rule set. First, any ambiguous locations were placed on the pending list until all other locations were processed. This ensured that we have the most complete context possible, and that all non-ambiguous locations were in the tree before the ambiguous locations were processed.

Once we finished processing all the non-ambiguous locations in the candidate list, we had a location tree, and a set of pending locations, some of which could fit more than one place on the tree. As each node is inserted into the tree, a running count of nodes and repeated nodes are kept. Using this information, when each ambiguous location was considered for insertion, a weighting was placed on each possible node in the tree that could serve as the parent node for the location. As an example, we consider the location "Cambridge". This could either be Cambridge, MA, USA, or Cambridge, UK. The tree shown in Figure 2, demonstrates a pair of possible parent leaf nodes to "Cambridge", with the occurrence count for each node in the article text. The location "Cambridge" can be added underneath either leaf node in the tree. The weight calculation shown in Equation 1 determines the correct node. The weight for node $n$ is determined by summing the insertion count for all the parent nodes of $n$ in the tree (up to three parents), and dividing by the total insertion count for the tree. A parent node is set to zero if the tree does not contain that node. The insertion count for each node is the total number of occurrences of the location represented by that node in the original article, so the insertion count for the whole tree is equal to the number of all location occurrences in the article.

$$
\text { Weight }_{n}=\frac{\left(C t_{\text {country }}+C t_{\text {state }}+C t_{\text {city }}\right)}{C t_{\text {total }}}
$$

Calculating weight for the two possible parent nodes

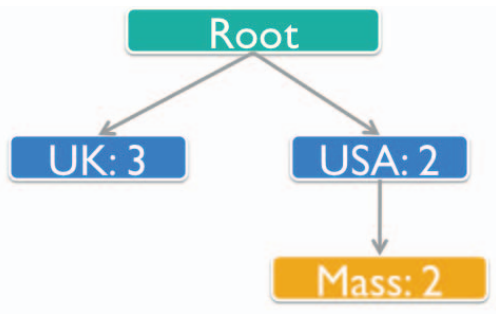

Figure 2. Example Location Tree Showing Duplicated Node Count

\section{Table 5. Raw SVM and Resolved Geospatial NE Results}

\begin{tabular}{||l|l|l|l||}
\hline & Precision & Recall & F-Measure \\
\hline Raw Results & 0.510 & 0.998 & 0.675 \\
\hline Resolved Results & 0.869 & 0.789 & 0.820 \\
\hline
\end{tabular}

of "Cambridge", we use 3 as the insertion count for UK, and 4 for the insertion count for Massachusetts, 2 from the Massachusetts node and 2 from the USA node. Dividing both by the insertion count of 7 for the entire tree, we find Weight $_{U K}=0.428$ and Weight $_{M A}=0.572$. As the Weight MA $_{\text {is }}$ ine higher of the two, "Cambridge" is associated with Cambridge, MA.

The equation for the weight calculation was chosen based on empirical testing. Our testing showed that we place $78 \%$ of the ambiguous locations correctly in the tree. This does not factor in unambiguous locations, as they are always placed correctly in the tree.

Figure 3 shows the complete location tree for the Operation Nickel Grass Wikipedia article. Note that the Operation Orchard node is the only incorrect node placed in the tree.

\subsection{Results}

Table 5 shows the raw results from the SVM NE recognition along with the results after processing through geospatial resolution and disambiguation. While geocoding resolution increased the precision significantly, up 25\% from the raw SVM, it did so at the cost of recall, because some 


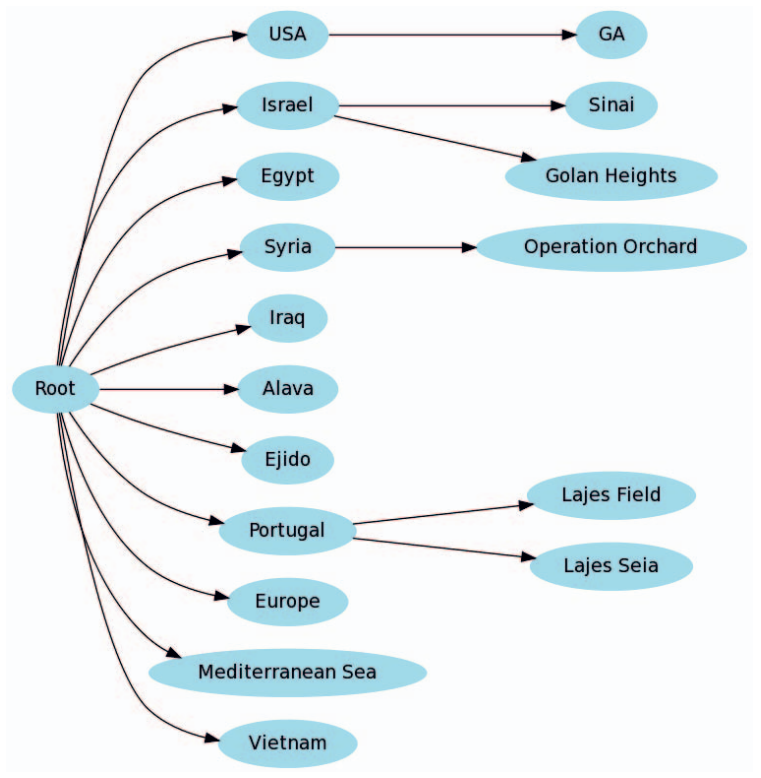

Figure 3. Complete Location Tree for the Operation Nickel Grass Wikipedia Article

of the actual geospatial NEs were filtered out. Across the Wikipedia corpus of 90 articles, we were able to successfully geocode $82 \%$ of the location NEs to a coordinate pair. The lowest f-measure was the Battle of Gettysburg article, with an f-measure of $68.2 \%$, up to the highest f-measure of 96.1\%, for the Dec. 7, 1941 attack on Pearl Harbor article (not shown in Figure 4).

Figure 4 shows a more detailed breakdown of the precision, recall, and f-measure for a subset of the articles processed. The word count is shown in parentheses after the article name. The results in this chart show, in general, the same trend shown by Table 3 . The Geografikos package was generally able to extract locations with a higher performance from articles that contain a higher percentage of non-ambiguous locations. The word count of the article did not appear to have any effect on the overall performance.

\subsection{Analysis of Results}

We encountered challenges with name resolution in the geocoder in some areas of the world. For a small set of locations where Google Geocoder returned errors, we were forced to manually return locations. Google Geocoder also returned US state and Canadian province names in a variety of formats. To account for this, we performed postprocessing on the Geocoder results. We found that the geocoder performed much better on land-based locations than ocean and sea-based. This is primarily because land tends to be better divided and named than the ocean. This problem found primarily in articles about naval battles, like the Battle of Midway article. We also found that Google Geocoder has much better data in its gazetteer for the US and Western Europe than other areas of the world. While we were able to resolve major cities in Asia and Africa, smaller-scale features were often returned as "not found". Russian and Eastern Asian locations also introduced the occasional problem of other character sets in Unicode characters. We found that the articles in our corpus did not refer to very many features at smaller than a city level in general, so these geocoding problems did not significantly impact our performance.

Historical location names caused some small issues with the geocoder, but our research demonstrated that, for North America and Europe at least, Google Geocoder has historical names in its gazetteer going back at least 300 years.

Finally, we found that we also had problems with the context-based disambiguation in North America in areas where the city and feature names have significant overlap. The ultimate result of this was that places with globally unique city and feature names performed the best, as there was no need to run the context disambiguation algorithms. We found that the worst areas in North America are the Northeast, and the Southwest US where there are significant overlap between place names in the US and place names in Mexico. In general, our context disambiguation algorithm performed at $78 \%$, but in these areas of the country, the performance dropped to around $64.5 \%$, due to the significant place name overlap.

\section{Conclusions and Future Work}

We continue to enhance the Geografikos system to allow searches for text content to be filtered by geographic area, through a search in two steps. The first would be a standard free text search on Wikipedia, for articles about the topic. That list could then be further filtered to those articles with appropriate locations. Reversing this paradigm, the location data provided by the Geografikos package could also allow location-centric search. If a user were about to go on vacation to Beijing, China, they could be presented with an interface allowing them to select an area around Beijing, and the system could return all articles that have locations that fall into that bounding area.

Furthermore, this database of locations could enable the visualization of Wikipedia articles through a geospatial interface. For instance, consider an interface that would allow a user to select a Wikipedia article, and then present the user with a map of all the locations from the article. Each location on the map would be clickable, and provide the sentences or paragraph around that NE from the text. Imagine putting "World War II" into this interface, and being presented with a map of Europe, Africa, and the Pacific theatre, with all the locations from the article marked and 


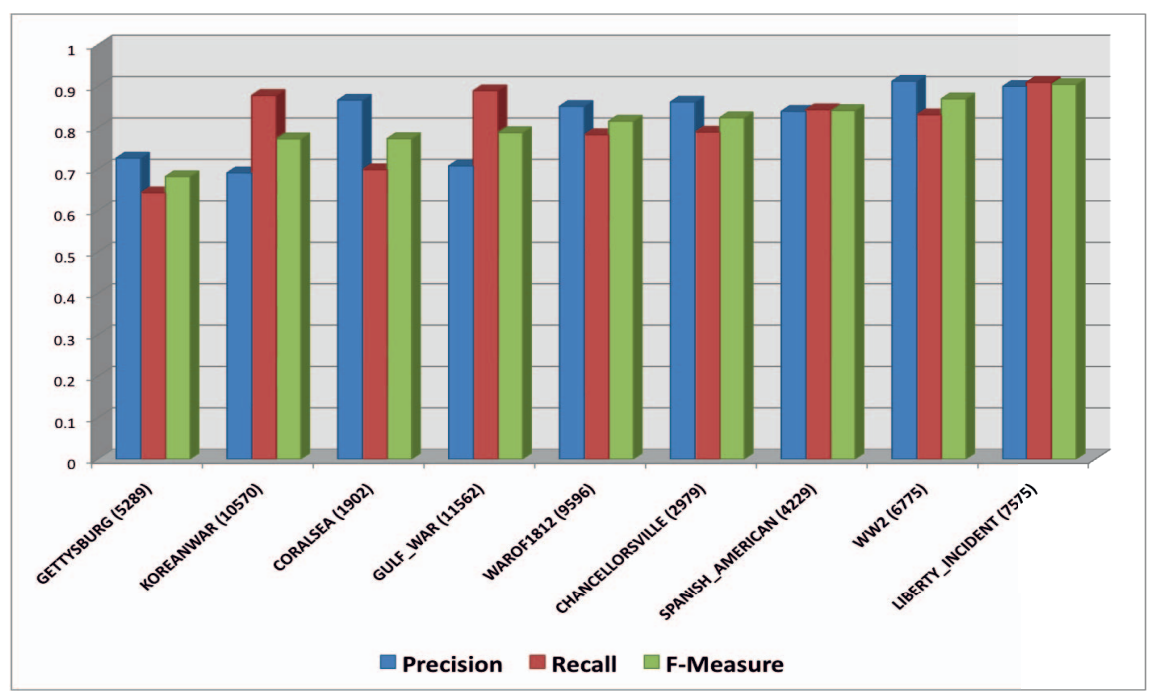

Figure 4. Detailed Results for a Subset of Wikipedia Articles

clickable. This kind of visualization would be an excellent teaching tool, and possibly reveal implicit information and relationships that are not apparent from the text of the article. Applied to other corpuses of information, this kind of information could also be very useful in finding geospatial trends and relationships. For instance, consider a database of textual disease outbreak reports. The Geografikos package could extract all the locations, allowing graphical presentation on a map, allowing trends to be found much more easily. With additional work, the geospatial data extracted by the Geografikos package could be combined with temporal information. While many of these visualization tools already exist, they are driven from structured databases. Our overall contribution in this area is the generation of the article and geospatial entity association database to drive these tools, through our novel data structure and algorithm for resolution of ambiguous geospatial NEs based on article context. This is just a small set of the possibilities for increased capability in geospatial information retrieval provided by associating a structured list of geospatial entities with a free text corpus.

\section{References}

[1] A. Bhole, B. Fortuna, M. Grobelnik, and D. Mladenić. Extracting Named Entities and Relating Them over Time Based on Wikipedia. Informatica, 22(4):20-26, 2007.

[2] S. Cucerzan. Large-scale named entity disambiguation based on Wikipedia data. EMNLP, 2007.

[3] W. Dakka and S. Cucerzan. Augmenting wikipedia with named entity tags. IJCNLP, 2008.

[4] T. D'Roza and G. Bilchev. An Overview of Location-Based Services. BT Technology Journal, 21(1):20-27, 2003.

[5] R. Grishman and B. Sundheim. MUC-6: A brief history. In ICCL, pages 466-471. ACL, 1996.
[6] M. Habib. Improving scalability of support vector machines for biomedical named entity recogntion. Ph.D. Dissertation, CS Department, UCCS, 2008.

[7] H. Isozaki and H. Kazawa. Efficient support vector classifiers for named entity recognition. In $I C C L$, pages $1-7$, Morristown, NJ, USA, 2002. ACL.

[8] K.-J. Lee, Y.-S. Hwang, and H.-C. Rim. Two-phase biomedical ne recognition based on svms. In $A C L$, pages 33-40, Morristown, NJ, USA, 2003. ACL.

[9] A. Mansouri, L. Affendey, and A. Mamat. Named entity recognition using a new fuzzy support vector machine. IJCSNS, 8(2):320, 2008.

[10] B. Martins, H. Manguinhas, and J. Borbinha. Extracting and Exploring the Geo-Temporal Semantics of Textual Resources. In IEEE ICSC, pages 1-9, 2008.

[11] V. Sehgal, L. Getoor, and P. Viechnicki. Entity resolution in geospatial data integration. In ACM int. sym. on Advances in GIS, pages 83-90. ACM, 2006.

[12] D. Smith and G. Crane. Disambiguating geographic names in a historical digital library. Lecture Notes in CS, pages 127-136, 2001.

[13] K. Takeuchi and N. Collier. Use of support vector machines in extended named entity recognition. In COLING-02, pages 1-7, Morristown, NJ, USA, 2002. ACL.

[14] V. Vapnik. The Nature of Statistical Learning Theory. Springer, 2000.

[15] Ø. Vestavik. Geographic Information Retrieval: An Overview, 2003.

[16] C. Wang, X. Xie, L. Wang, Y. Lu, and W. Ma. Detecting geographic locations from web resources. 2005 workshop on GIR, pages 17-24, 2005.

[17] J. Witmer and J. Kalita. Mining Wikipedia Article Clusters for Geospatial Entities and Relationships. AAAI Spr. Sym., 2009.

[18] W. Zong, D. Wu, A. Sun, E. Lim, and D. Goh. On assigning place names to geography related web pages. In ACM/IEEECS joint conf. on Digital libraries, pages 354-362. ACM, 2005. 